\title{
OPEN A mutation in transmembrane protein 135 impairs lipid metabolism in mouse eyecups
}

\author{
Michael Landowski $i^{1,2,5}$, Vijesh J. Bhute ${ }^{1,3,5}$, Tetsuya Takimoto ${ }^{1}$, Samuel Grindel ${ }^{1}$, \\ Pawan K. Shahi ${ }^{1,2,4}$, Bikash R. Pattnaik ${ }^{1,2,4}$, Sakae Ikeda ${ }^{1,2}$ \& Akihiro Ikeda ${ }^{1,2}$
}

Aging is a significant factor in the development of age-related diseases but how aging disrupts cellular homeostasis to cause age-related retinal disease is unknown. Here, we further our studies on transmembrane protein 135 (Tmem135), a gene involved in retinal aging, by examining the transcriptomic profiles of wild-type, heterozygous and homozygous Tmem135 mutant posterior eyecup samples through RNA sequencing (RNA-Seq). We found significant gene expression changes in both heterozygous and homozygous Tmem135 mutant mouse eyecups that correlate with visual function deficits. Further analysis revealed that expression of many genes involved in lipid metabolism are changed due to the Tmem135 mutation. Consistent with these changes, we found increased lipid accumulation in mutant Tmem135 eyecup samples. Since mutant Tmem135 mice have similar ocular pathologies as human age-related macular degeneration (AMD) eyes, we compared our homozygous Tmem135 mutant eyecup RNA-Seq dataset with transcriptomic datasets of human AMD donor eyes. We found similar changes in genes involved in lipid metabolism between the homozygous Tmem135 mutant eyecups and AMD donor eyes. Our study suggests that the Tmem135 mutation affects lipid metabolism as similarly observed in human AMD eyes, thus Tmem135 mutant mice can serve as a good model for the role of dysregulated lipid metabolism in AMD.

The most significant risk factor for age-related diseases is aging itself. However, how aging is associated with age-related disease at the molecular level is still largely to be understood. The polygenic nature of age-related neurodegenerative diseases such as age-related macular degeneration (AMD) and Alzheimer's disease makes identification of the molecular pathways involved more challenging ${ }^{1,2}$.Therefore, mouse mutants showing agerelated symptoms are valuable for isolating genetic factors that contribute to aging and age-related diseases.

We identified one such mouse strain harboring a mutation in transmembrane protein 135 (Tmem$135^{\text {FUN025/FUN025 }) ~ t h a t ~ d i s p l a y s ~ s i g n s ~ o f ~ a c c e l e r a t e d ~ a g i n g ~ i n ~ t h e ~ r e t i n a ~ a s ~ w e l l ~ a s ~ p a t h o l o g i e s ~ o b s e r v e d ~ i n ~ A M D ~}$ including autofluorescent aggregates in the subretinal space, increased inflammation, and thickened retinal pigmented epithelium (RPE) at a young age ${ }^{3}$. These phenotypes indicated that Tmem 135 regulates retinal aging and its impairment results in age-related disease phenotypes. Tmem 135 encodes a 52 kilodalton protein with five transmembrane domains that share similarities with the Tim 17 protein family, a group of proteins important for mitochondria biogenesis ${ }^{3,4}$. TMEM135 was originally identified as a gene associated with longevity and cold stress in C. elegans ${ }^{5}$. Previous studies also suggested the function of TMEM135 in lipid metabolism ${ }^{5,6}$. In addition, a study using transgenic mice overexpressing the Tmem135 gene (Tmem135 TG) showed that Tmem135 overexpression results in RPE dysmorphogenesis and degeneration ${ }^{7}$. Electron micrographs of the RPE from Tmem 135 $5^{\text {UNO25/FUN025 }}$ mice showed over-fused (elongated) mitochondrial networks while the RPE of Tmem 135 TG mice displayed over-fragmented mitochondrial networks relative to controls ${ }^{3,7}$. Despite their morphological differences, both Tmem135 $5^{\text {FUNO25/FUN025 }}$ and Tmem 135 TG RPE show reduced mitochondrial function ${ }^{8}$. Taken together, these results strongly indicated that TMEM135 acts as a pro-mitochondrial fission factor and regulates "mitochondrial dynamics," the collective term for the fusion, fission, and mitophagy events mitochondria undergo to preserve their shape, number and function" ${ }^{9}$, which is critical for RPE health. One striking age-

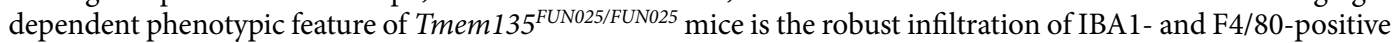
immune cells into the subretinal space ${ }^{3}$. Accumulation of subretinal immune cells has been associated with

\footnotetext{
${ }^{1}$ Department of Medical Genetics, University of Wisconsin-Madison, Madison, WI, USA. ${ }^{2}$ McPherson Eye Research Institute, University of Wisconsin-Madison, Madison, WI, USA. ${ }^{3}$ Department of Chemical Engineering, Imperial

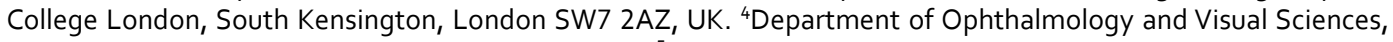
University of Wisconsin-Madison, Madison, WI, USA. ${ }^{5}$ These authors contributed equally: Michael Landowski and Vijesh J. Bhute. ${ }^{\varpi}$ email: aikeda@wisc.edu
} 
all stages of AMD and believed to contribute to the chronic inflammation underlying AMD. The phenotypic similarity between Tmem $135^{\text {FUNO25/FUNO25 }}$ mice and human AMD motivates us to identify molecular pathways underlying the retinal pathologies in Tmem $135^{\text {FUN025/FUN025 mice. }}$

In this study, we performed RNA sequencing (RNA-Seq) of the eyecup (RPE/choroid/sclera) of Tmem-

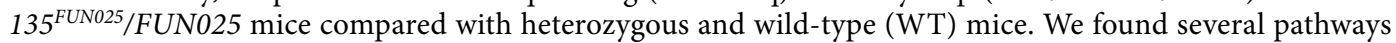
including cholesterol metabolism, fatty acid metabolism, and steroid metabolic processes were significantly upregulated in Tmem $135^{\text {FUN025/FUN025 }}$ mice. We observed increased lipid accumulations in Tmem 135 FUN025/FUN025 eyecups, which confirms the changes in lipid metabolism. Some of these gene expression changes overlap with those previously observed in human AMD-afflicted RPE/choroid samples. Our study suggests that lipid metabolism is a molecular pathway affected by the Tmem 135 mutation as observed in human AMD eyes, thus Tmem135 mutant mice can serve as a good model for the role of dysregulated lipid metabolism in AMD.

\section{Results}

Heterozygous and homozygous Tmem135 mutant mice display ocular phenotypes. A mutation in Tmem 135 effects retinal homeostasis in mice ${ }^{3,7}$. Homozygous Tmem 135 mutant (Tmem135 ${ }^{\text {FUN025/FUN025) }}$ mice show early-onset of aging-associated retinal changes with accelerated progression and pathologies similar to those observed in AMD as early as 2 months of age ${ }^{3}$. At that age, heterozygous Tmem135 mutant (Tmem$135^{\mathrm{FUNO} 25 /+}$ ) mice did not show any ocular abnormalities (data not shown). Here, we investigated whether heterozygous Tmem 135 mutant (Tmem $135^{\text {FUN025/+ }}$ ) mice display ocular abnormalities at 12 months of age. No distinct morphological changes were observed in Tmem $135^{\text {FUNO25/+ }}$ retinal sections compared to WT retinal sections (Fig. 1A). This contrasts the presence of photoreceptor degeneration and subretinal immune cells present in homozygous Tmem135 mutant (Tmem135 13 NO25/FUN025) retinas (Fig. 1A). We quantitated photoreceptor degeneration in Tmem $135^{\text {FUNO25/FUN025 }}$ retinas by normalizing the length of the outer nuclear layer (ONL) to the inner nuclear layer (INL) to calculate the ONL thickness (ONLT) ratio ${ }^{3}$. While there were no ONLT differences between WT and Tmem $135^{\mathrm{FUNO} 25 /+}$, the ONLT ratios of both groups were significantly higher than those measured for the Tmem135 13 NO25/FUN025 retinas (Fig. 1B). Also, there were no differences in the thickness

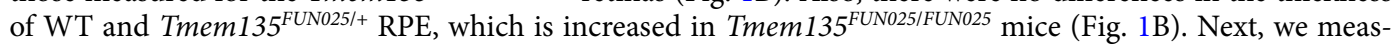
ured visual function through electroretinography (ERG). 12-month-old Tmem135 $5^{\text {FUN025/+ }}$ mice had significantly smaller scotopic a-waves than WT mice except at the $30 \mathrm{~cd}^{*} \mathrm{~s} / \mathrm{m}^{2}$ flash intensity (Fig. 1C). The scotopic b-waves of Tmem $135^{\mathrm{FUNO} 25 /+}$ mice were significantly decreased at $0.03,1,3$, and $10 \mathrm{~cd}^{*} \mathrm{~s} / \mathrm{m}^{2}$ flash intensities relative to WT mice (Fig. 1D). No changes in the photopic a-wave, photopic b-wave, and c-wave were noted between WT and Tmem $135^{\text {FUNO25/+ }}$ mice (1E-G). Given that scotopic ERG responses and not photopic ERG responses were changed, rods are sensitive to the heterozygosity of the Tmem135 mutation. The scotopic a-waves and b-waves at all intensities as well as photopic a-wave, photopic b-wave, and c-wave were significantly attenu-

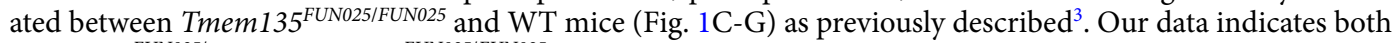

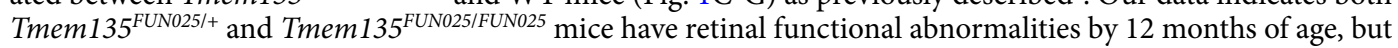
only Tmem $135^{\mathrm{FUN} 025 / F U N 025}$ mice have pronounced retinal morphological differences compared to WT mice.

Mutation in Tmem135 results in widespread transcriptional changes. To identify the origin of the retinal abnormalities due to the Tmem 135 mutation, we performed RNA-Seq on eyecups collected from

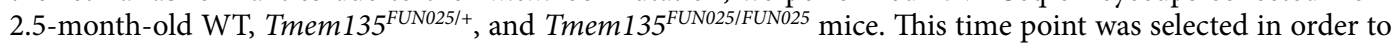
examine transcriptional changes in the early stage of the pathological changes in Tmem $135^{\text {FUN025/FUN025 }}$ mice rather than later stages that would be affected by extensive retinal degeneration (Fig. 1$)^{3}$. These eyecup tissue preparations include the retinal pigmented epithelium (RPE), a cell-type particularly sensitive to the levels of functional TMEM $135^{7}$ and postulated as the primary site affected by the Tmem $135^{\text {FUNO25 }}$ mutation $^{3}$. Global tran-

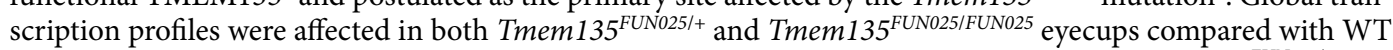
controls. 1723 genes and 1785 genes were significantly altered (adj.p value $<0.05$ ) in the Tmem135 ${ }^{\text {FUNO25/+ }}$ and Tmem $135^{\text {FUNO25/FUN025 }}$ genotypes relative to WT, respectively. 1112 were commonly affected between the Tmem$135^{\text {FUNO25/+ }}$ and Tmem $135^{\text {FUNO25/FUNO25 }}$ eyecups compared to WT. There were 611 and 673 genes uniquely changed in Tmem $135^{\text {FUN025/+ }}$ and Tmem $135^{\text {FUN025/FUN025 }}$ eyecups, respectively. We narrowed our RNA-seq analysis on genes with twofold expression differences in Tmem $135^{\text {FUNO25/+ }}$ and Tmem $135^{\text {FUNO25/FUN025 }}$ eyecups (Fig. 2A,B). In Tmem $135^{\text {FUNO25/+ }}$ eyecups, there were 277 and 418 genes that had twofold upregulation and downregulation compared to WT, respectively (Fig. 2A). Similarly, there were 333 genes that had twofold upregulation and 433

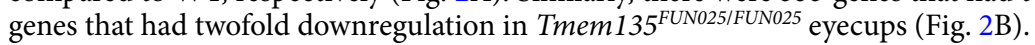

Next, we conducted gene set enrichment analysis (GSEA) to identify potential pathways which are affected due to the Tmem 135 mutation. Several pathways were significantly downregulated (FDR $<0.01$ ) in both Tmem$135^{\text {FUN025/+ }}$ and Tmem $135^{\text {FUNO25/FUN025 }}$ as compared to WT including contractile fiber, sensory perception of light stimulus, ribosome, mitochondrial membrane part, Parkinson disease, and synaptic vesicle cycle (Table 1 and Fig. 2C). On the other hand, several pathways including cholesterol metabolism, fatty acid metabolism, and steroid metabolic processes were significantly upregulated $(\mathrm{FDR}<0.01)$ exclusively in Tmem $135^{\text {FUN025/FUN025 }}$ eyecups relative to WT (Table 1 and Fig. 2D). Importantly, none of the pathways were significantly upregulated $($ FDR $<0.01)$ between Tmem $135^{\text {FUNO25/+ }}$ and WT eyecups (Table 1). Our results show there are distinct pathways disrupted by the Tmem 135 mutation in the murine eyecup.

Transcriptomic differences between heterozygous and homozygous Tmem135 mutant mice. To further elucidate important pathways perturbed by the Tmem 135 mutation, we undertook a differential analysis focusing on the Tmem $135^{\mathrm{FUNO25/+}}$ and Tmem $135^{\mathrm{FUNO25/FUNO25}}$ genotypes. There were 84 genes which showed significant differences (adj. $p<0.05$ ), and 27 of these genes had more than two-fold expression 
A)

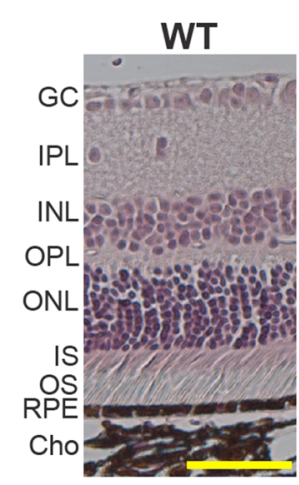

C)

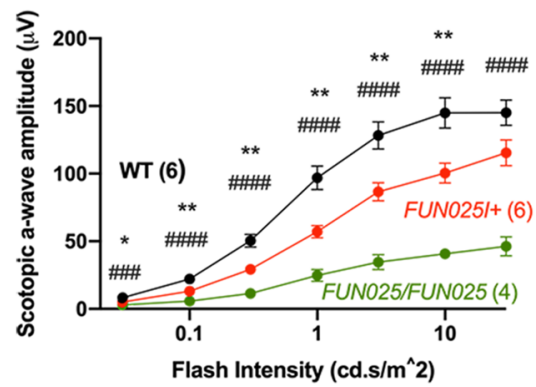

E)

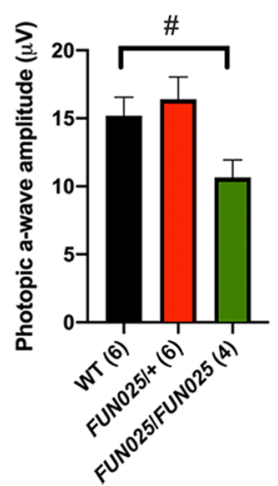

B)
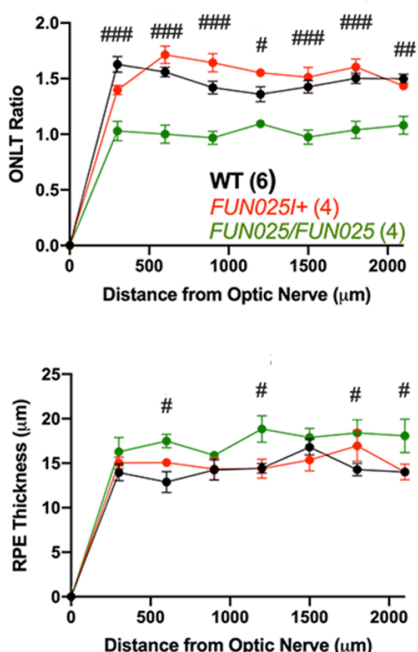

D)

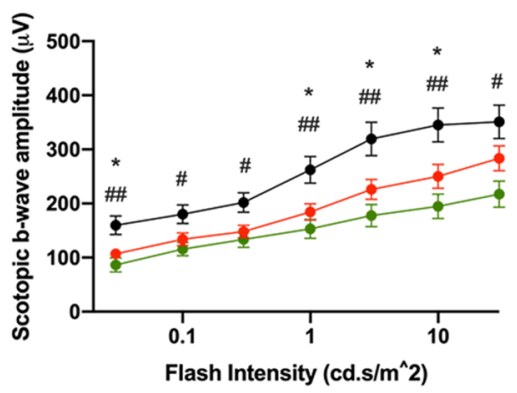

G)
F)

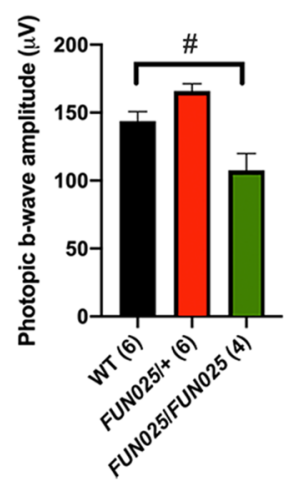

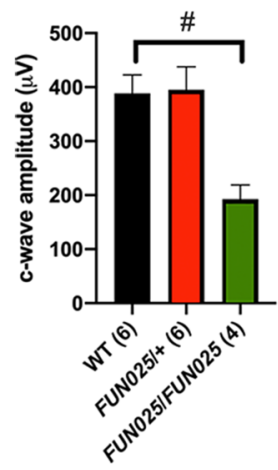

Figure 1. Retinal Phenotype of 12-Month-Old Tmem $135^{\mathrm{FUN} 025} /^{+}$mice. (A) Representative images of the

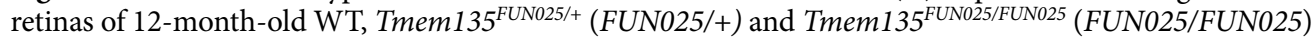
mice. Note the decreased ONL thickness and subretinal immune cell (denoted with a yellow asterisk) in the 12-month-old Tmem $135^{\text {FUN025/FUN025 }}$ retina which are not present in the 12-month-old Tmem135 ${ }^{\text {FUN025/+ }}$ mice. Magnification $=20 \times$. Scale bar $=100$ microns. $($ B $)$ ONLT and RPE ratios. No significant differences were noted between the WT and Tmem135 ${ }^{\text {FUN025/+ }}$ mice. (C-G) ERG recordings for 12-month-old WT, Tmem135 $5^{\text {FUN025/+ }}$

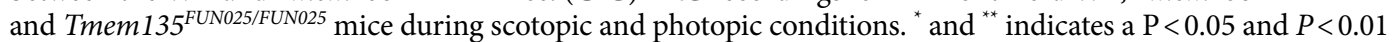
significance by post hoc Tukey test following a significant difference detected by one-way ANOVA between WT and Tmem135 FUN025/+. \#, \#\#, \#\#\#, and ${ }^{\# \# \#}$ indicates a $\mathrm{P}<0.05, \mathrm{P}<0.01, \mathrm{P}<0.001$, and $P<0.0001$ significance by post hoc Tukey test following a significant difference detected by one-way ANOVA between WT and

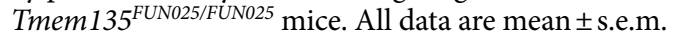

changes. 5 of these genes had twofold decreased expression whereas 22 genes had twofold increased expression

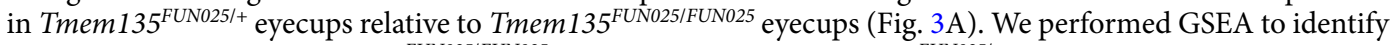
pathways enriched in Tmem $135^{\text {FUNO25/FUN025 }}$ eyecups relative to Tmem $135^{\text {FUNO25/+ }}$ eyecups. There was significant enrichment of cholesterol metabolism $(\mathrm{NES}=2.32$, $\mathrm{FDR}=0.013)$ (Fig. $3 \mathrm{~B})$, peroxisome proliferator-activated receptor $(\mathrm{PPAR})$ signalling $(\mathrm{NES}=2.28, \mathrm{FDR}=0.011)($ Fig. $3 \mathrm{C})$, and complement and coagulation cascades $(\mathrm{NES}=2.18, \mathrm{FDR}=0.04)$ (Fig. 3D) in Tmem $135^{\text {FUN025/FUN025 }}$ eyecups when compared with Tmem $135^{\mathrm{FUN025/+}}$ eyecups. These genes were unchanged between Tmem $135^{\mathrm{FUNO25/+}}$ and WT eyecups (Fig. 3B,D). Our results impli- 
A)
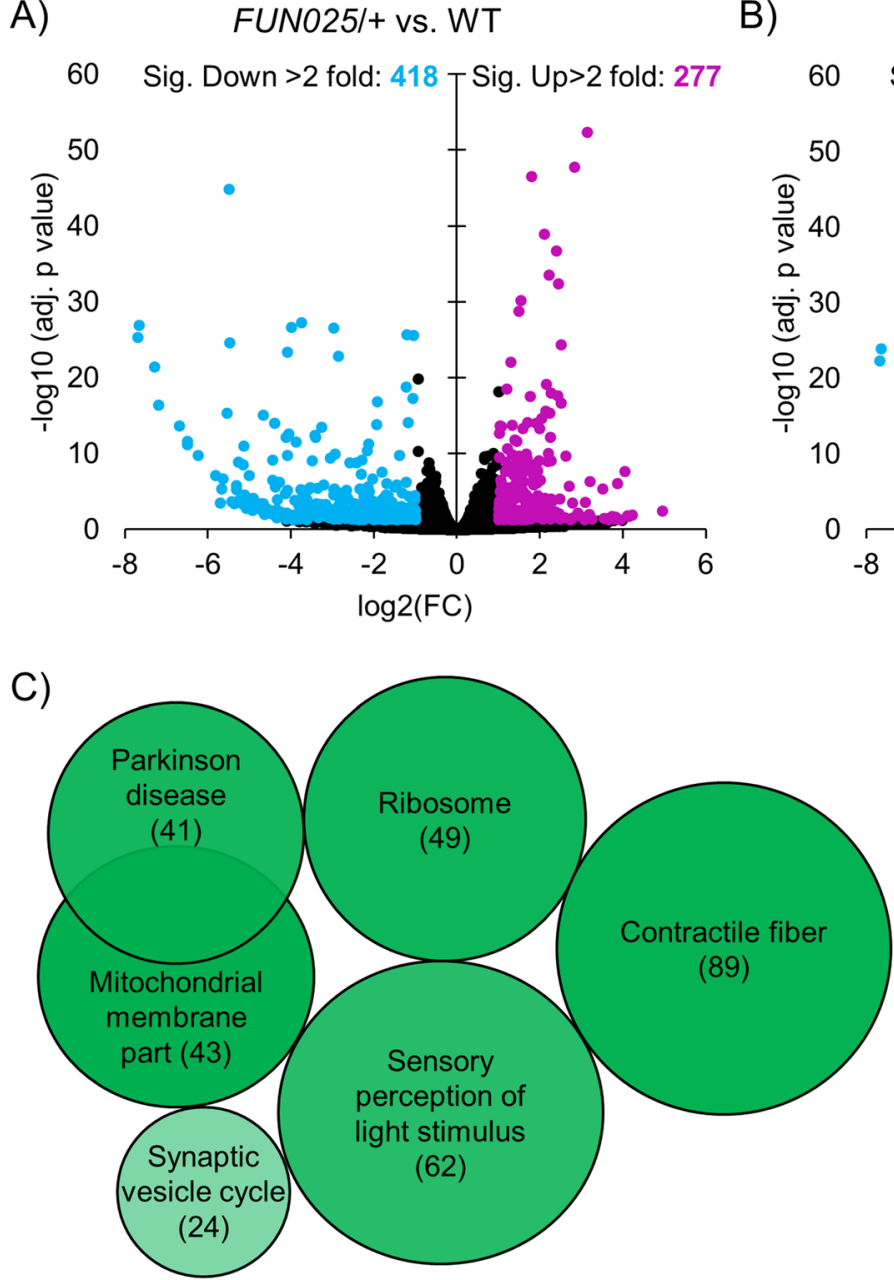

B)

\section{FUN025/FUN025 vs. WT}

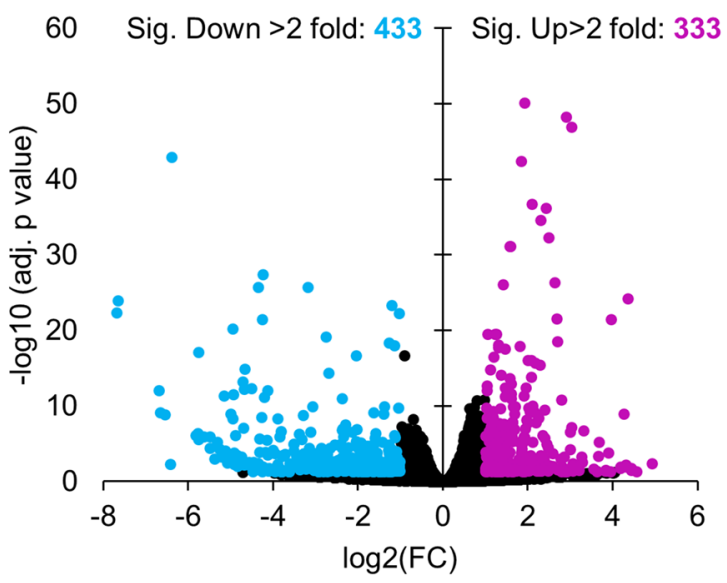

Cholesterol
metabolism (27)

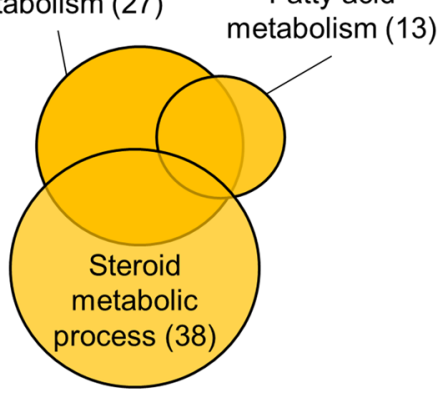

Significant positively enriched pathways exclusively in FUNO25/FUNO25 vs. WT (FDR<0.01)

Significant negatively enriched pathways in both FUNO25/+ vs WT and FUNO25/FUNO25 vs. WT (FDR<0.01)

Figure 2. RNA-Seq analysis of 2.5-month-old heterozygous and homozygous Tmem 135 mutant eyecups compared to WT eyecups. Volcano plot highlighting differentially-expressed genes with fold changes greater than 2 that reach statistical significance $(p<0.05)$ between $(A)$ Tmem135 1 FUN025/+ $(F U N 025 /+)$ and WT eyecups as well as (B) Tmem135 FUN025/FUN025 (FUN025/FUN025) and WT eyecups. Genes that are upregulated and downregulated are highlighted in magenta and cyan, respectively. Gene Set Enrichment Analysis (GSEA) results of significantly enriched gene sets $(\mathrm{FDR}<0.01)$ of $(\mathbf{C})$ downregulated genes and $(\mathbf{D})$ upregulated in

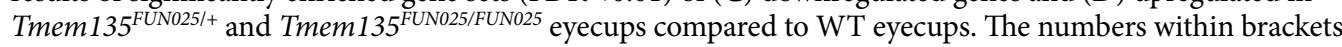
indicate the number of genes in the leading edge in the gene set and the colour indicates the normalized enrichment score where the lighter tone means less enrichment and darker tone means more enrichment.

cate cholesterol metabolism, PPAR signalling and complement and coagulation cascades as important pathways

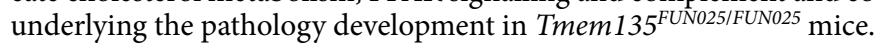

Correlations between differential gene expression and number of Tmem135 mutant alleles. We determined if any of the genes with differential expression correlated with the number of Tmem 135 mutant alleles. 72 genes had significant correlations (FDR $<0.01$ ) with the Tmem135 mutation status of mice where 35 genes were downregulated and 37 genes were upregulated in Tmem135 $5^{\text {FUN025/FUN025 eyecups }}$ when compared with WT eyecups (Fig. 4A, B). We used signalling network analysis to identify potential mechanisms which might be affected in a mutation dependent manner. We identified BCL6 corepressor (Bcor) which had a negative correlation score of -0.9 . BCOR can repress the transcription of target genes by interacting with histone deacetylases (HDACs) (Fig. 4C) and non-canonical polycomb recessive complex 1 (PRC1) that can downregulate peroxisome proliferator activated receptor gamma (Pparg) expression (Fig. 4D). We also observed a very strong positive correlation of 0.98 for isocitrate dehydrogenase 1 (Idh1) which was significantly increased in Tmem 135 $5^{\text {FUN025/FUN025 }}$ mice. Idh1 expression can be regulated by FOXO transcription factors and sterol regulatory element binding proteins (SREBFs) (Fig. 4E), both of which play important roles in lipid metabolism ${ }^{10,11}$. This analysis reveals a set of genes whose expression is correlated with the amount of functional TMEM135 and connected with transcriptomic pathways disrupted by the Tmem135 mutation. 


\begin{tabular}{|l|l|l|l|l|}
\hline \multirow{2}{*}{ Description } & \multicolumn{2}{l|}{$\begin{array}{l}\text { Tmem 135 } \\
\text { vs WUN025/+ }\end{array}$} & \multicolumn{2}{l|}{$\begin{array}{l}\text { Tmem 135 } \\
\text { IFUN025 }\end{array}$ vs WT } \\
\cline { 2 - 6 } & NES & FDR & NES & FDR \\
\hline WP4346: Cholesterol metabolism & & & 2.71 & 0 \\
\hline mmu01212: Fatty acid metabolism & & & 2.53 & 0 \\
\hline GO:0,008,202: Steroid metabolic process & & & 2.33 & 0.0023 \\
\hline mmu04721: Synaptic vesicle cycle & -1.78 & 0.0225 & -1.88 & 0.0058 \\
\hline GO:0,050,953: Sensory perception of light stimulus & -2.11 & 0.0009 & -1.97 & 0.0141 \\
\hline mmu05012: Parkinson disease & -2.17 & 0 & -2.01 & 0.0012 \\
\hline mmu03010: Ribosome & -2.21 & 0 & -2.03 & 0.0018 \\
\hline GO:0,043,292: Contractile fiber & -2.22 & 0 & -2.06 & 0 \\
\hline GO:0,044,455: Mitochondrial membrane part & -2.13 & 0 & -2.1 & 0 \\
\hline
\end{tabular}

Table 1. Pathway analysis using Gene Set Enrichment Analysis on Tmem135 $5^{\mathrm{FUNO25/+}}$ and Tmem $135^{\mathrm{FUN} 025 / \mathrm{FUN} 025}$ compared to WT gene sets ${ }^{*}{ }^{\star} \mathrm{FDR}$ cut-off of 0.01 is used for analysis. Empty cells indicate FDR was greater than 0.01 .

A)

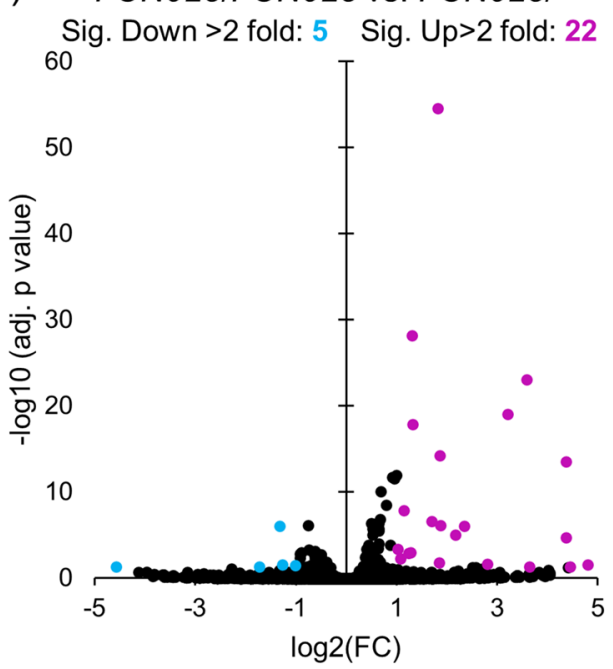

C)

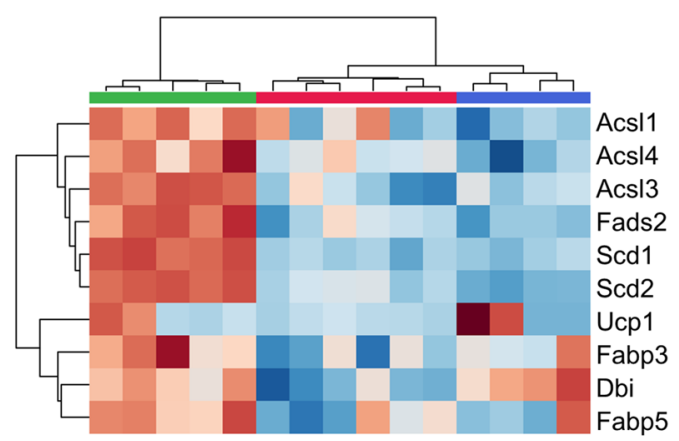

B) WP4346: Cholesterol Metabolism

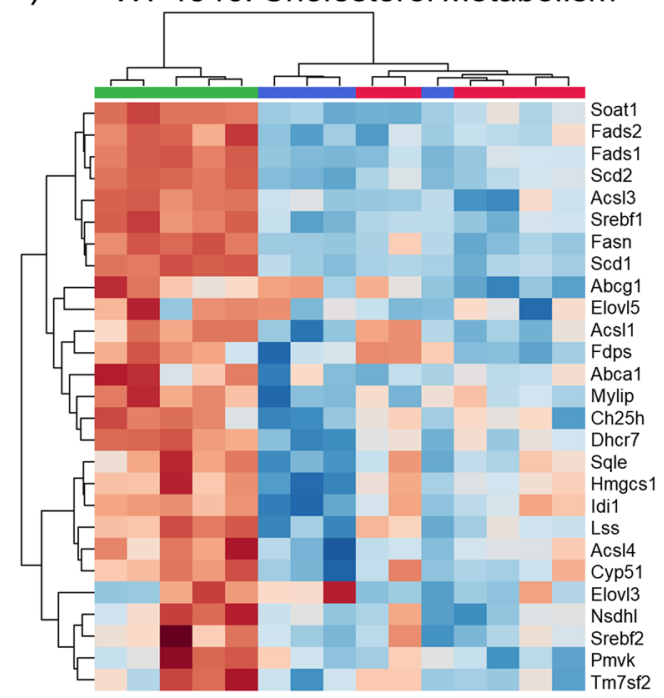

D) WP449: Complement and Coagulation Cascades

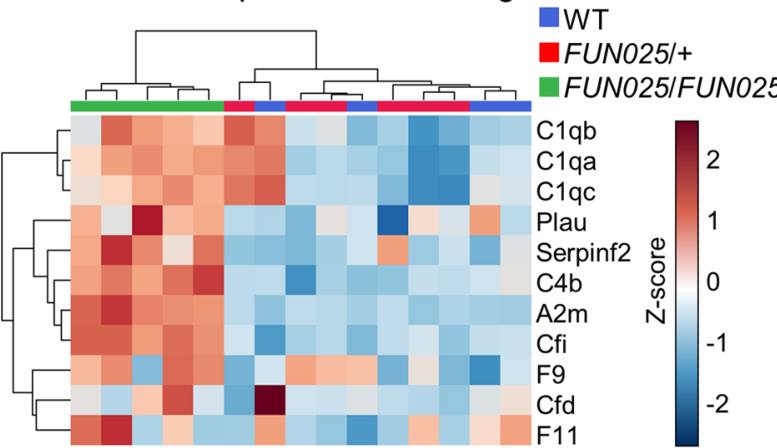

Figure 3. RNA-Seq analysis of 2.5-month-old homozygous Tmem 135 mutant eyecups compared to heterozygous Tmem 135 mutant eyecups. (A) Volcano plot highlighting differentially-expressed genes with fold changes greater than 2 that reach statistical significance $(p<0.05)$ between Tmem135 FUN025/FUN025 (FUN025/ FUN025) and Tmem $135^{\mathrm{FUN025/+}}$ (FUN025/+) eyecups. Genes that are upregulated and downregulated are highlighted in magenta and cyan, respectively. Heatmaps of differentially-expressed genes in the leading edge

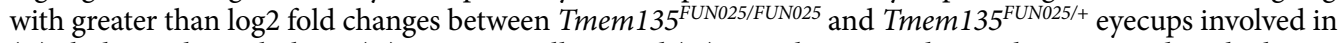
(B) cholesterol metabolism, (C) PPAR signalling, and (D) complement and coagulation cascades which were identified using gene set enrichment analysis. These genes were unchanged between Tmem $135^{\text {FUN025/+ }}$ and WT eyecups. 
A) Genes positively correlated with $T m e m 135$ mutation status

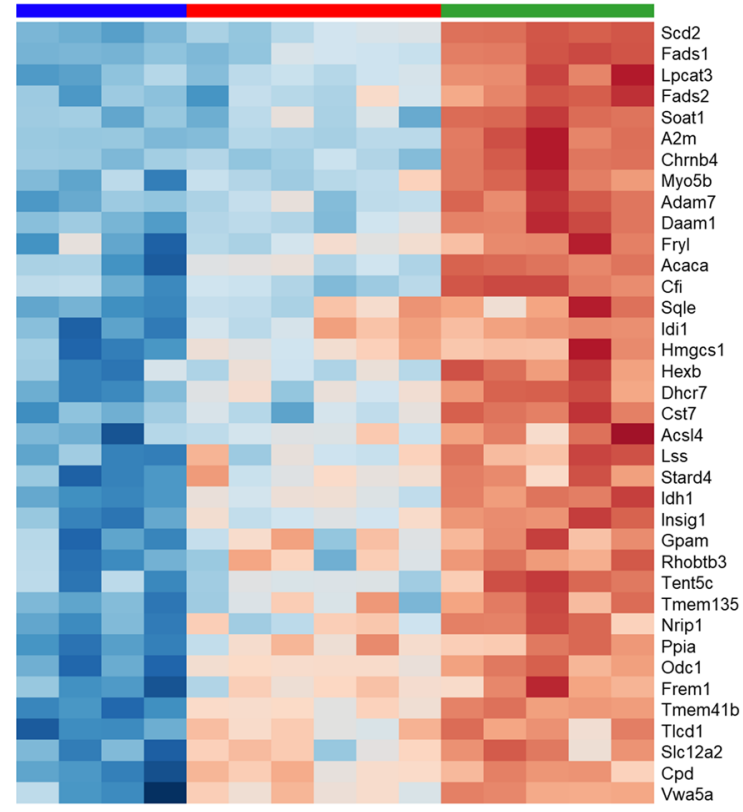

B) Genes negatively correlated with Tmem135 mutation status

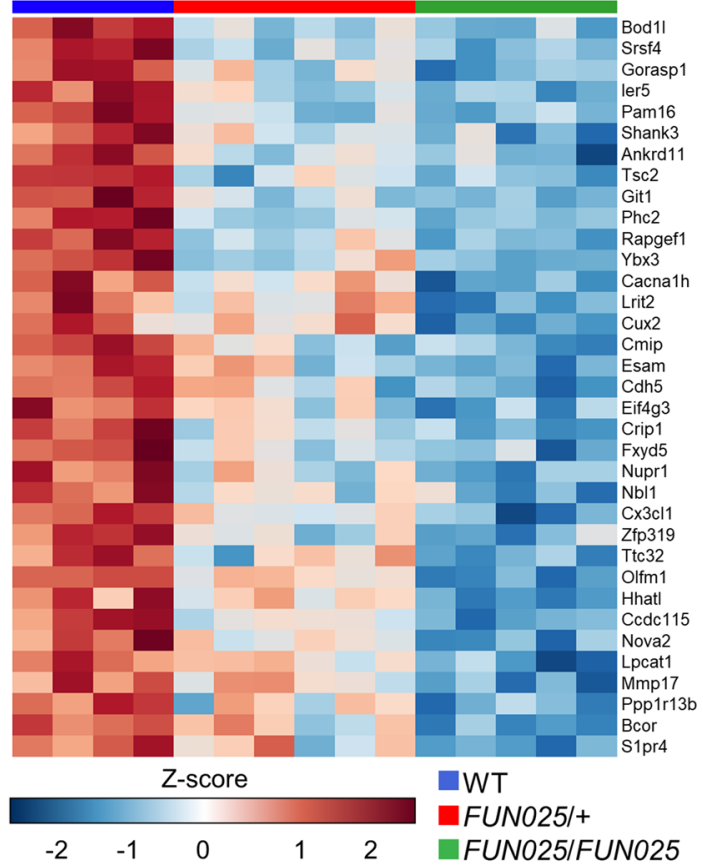

C)

\section{Bcor signalling interactions}

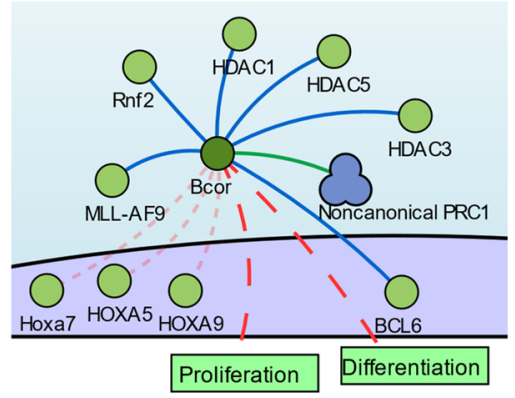

D) Non-canonical PRC1 signalling interactions

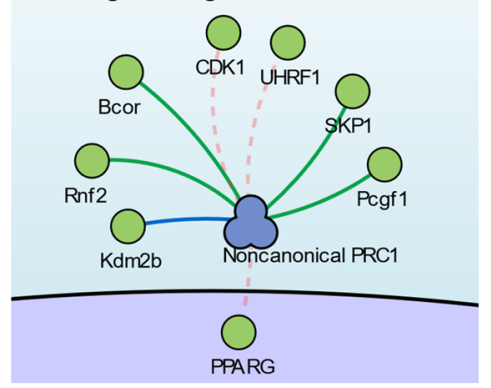

E)

\section{Idh1 signalling interactions}

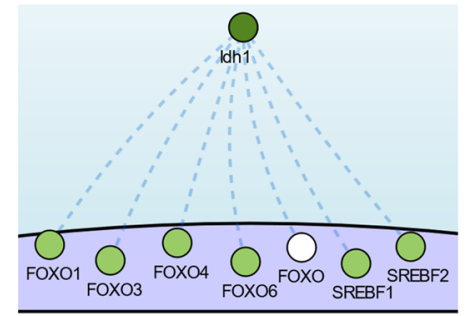

\begin{tabular}{|lll|}
\hline 0 & Protein & Up-regulate \\
0 & Protein family & Down-regulate \\
$\square$ & Phenotype/Stimulus & Physical interaction \\
0 & Complex & Direct \\
$\square$ & Nucleus & - Indirect \\
& & - Binding \\
\hline
\end{tabular}

Figure 4. Correlation analysis highlights important signalling pathways affected by Tmem 135 mutations. Heatmap of genes which are significantly $(\mathbf{A})$ positively and $(\mathbf{B})$ negatively correlated $(\mathrm{FDR}<0.01)$ with the

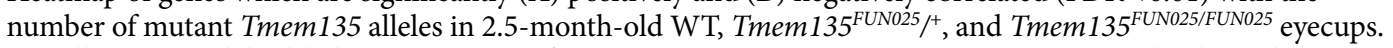
Signalling network highlights interactions of (C) BCL6 corepressor (Bcor), (D) non-canonical polycomb recessive complex 1 (PRC1), and (E) isocitrate dehydrogenase 1 (Idh1). Network generated using the SIGnalling Network Open Resource (SIGNOR 2.0).

Lipid levels in heterozygous and homozygous Tmem135 mutant mice. To validate our RNA-Seq results, we determined lipid levels within the eyecups of Tmem $135^{\text {FUNO25/+ }}$ and Tmem $135^{\text {FUN025/FUN025 mice. We }}$ prepared eyecup samples from 3-month-old and 12-month-old WT, Tmem $135^{\text {FUN025/+ }}$, and Tmem $135^{\text {FUN025/FUN025 }}$ mice to measure cholesterol concentrations. We detected significant increases of cholesterol in 3-month-old Tmem $135^{\text {FUNO25/FUNO25 }}$ eyecups $(21.97 \pm 1.45 \mu \mathrm{M} / \mathrm{mg})$ compared to WT $(15.85 \pm 4.35 \mu \mathrm{M} / \mathrm{mg})$ and Tmem$135^{\mathrm{FUN025/+}}$ eyecups $(14.37 \pm 1.94 \mu \mathrm{M} / \mathrm{mg}$ ) (Fig. $5 \mathrm{~A})$. Similar increases in cholesterol were observed in 12-monthold Tmem $135^{\text {FUNO25/FUN025 }}$ eyecups $(19.76 \pm 4.79 \mu \mathrm{M} / \mathrm{mg})$ relative to WT $(9.92 \pm 3.46 \mu \mathrm{M} / \mathrm{mg})$ and Tmem$135^{\mathrm{FUN} 025 /+}(12.49 \pm 4.38 \mu \mathrm{M} / \mathrm{mg})$ (Fig. $\left.5 \mathrm{~B}\right)$. We prepared RPE flat mounts from 3-month-old and 12-month-old 

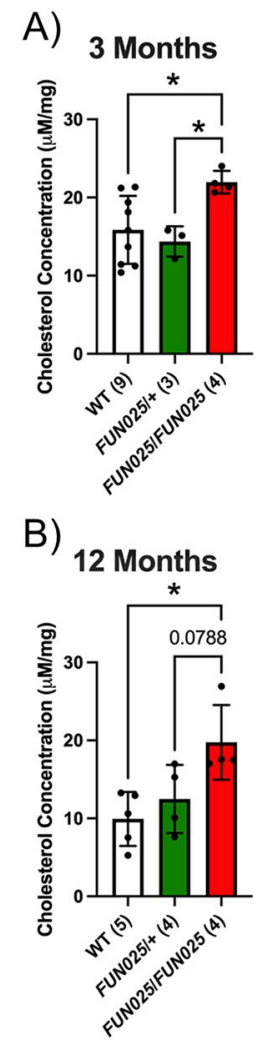

C)
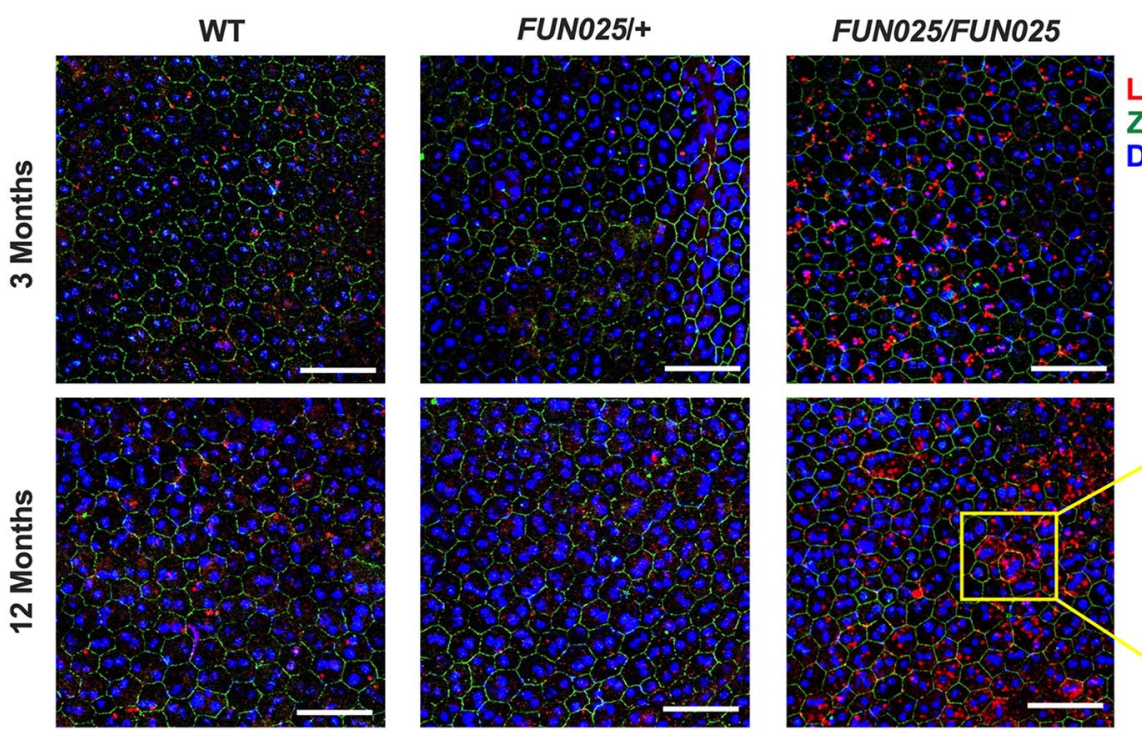

LipidTOX

ZO-1

DAPI

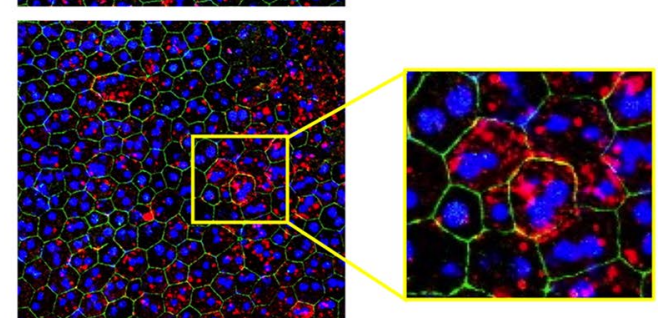

Figure 5. Lipid Levels in WT, Heterozygous and Homozygous Tmem135 Mutant Eyecups. Cholesterol levels in eyecups of (A) 3-month-old and (B) 12-month-old WT, Tmem135 $5^{\text {FUNO25/+ }}$ (FUN025/+), and Tmem135 FUN025/FUN025 (FUN025/FUN025) mice. * indicates a $P<0.05$ significance by post hoc Tukey test following a significant difference detected by one-way ANOVA. Numbers with brackets denote number of mice used for experiment. Each dot represents each individual sample. Data is presented as mean \pm s.d.. (C) Representative RPE flat mount images after labelling for tight junctions (green) and staining for neutral lipids (red) and nuclei (blue). Mag $=20 \times$, Scale Bar $=25$ microns.

WT, Tmem $135^{\mathrm{FUN} 025 /+}$, and Tmem $135^{\mathrm{FUN} 025 / \mathrm{FUN} 025}$ mice for staining with HSC LipidTox Red that detects neutral lipids and allows for their visualization. We detected an age-dependent increase of neutral lipid accumulations

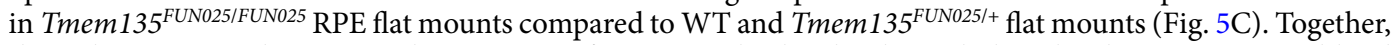
these data support the increased expression of genes involved in lipid metabolism by showing increased lipid accumulations in Tmem135 FUN025/FUN025 eyecups.

Lipid metabolic gene expression in eyecups and neural retinas. The RPE is a major tissue involved in lipid metabolism within the retina ${ }^{12}$. Since we detected significant lipid metabolic transcriptional changes from our RNA-seq study, we explored whether these changes were unique to the posterior eyecup containing the RPE. We prepared cDNA from RNA individually isolated from the posterior eyecup and neural retinas of

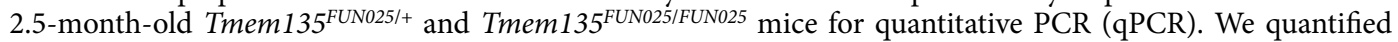
sterol regulatory element binding transcription factor 2 (Srebf2), Srebf1c, acetyl-CoA carboxylase alpha (Acaca), fatty acid synthase (Fasn), and stearoyl-CoA desaturase 1 ( $S c d 1)$. We found significant increases in all these genes in Tmem $135^{\text {FUNO25/FUNO25 }}$ eyecups compared to Tmem $135^{\text {FUNO25/+ }}$ eyecups (Fig. 6A). Strikingly, only Scd1 was upregulated in the neural retinas of Tmem $135^{\text {FUNO25/FUN025 }}$ mice relative to Tmem $135^{\text {FUN025/+ }}$ neural retinas (Fig. 6B). These data show that Tmem 135 mutant-induced increases of lipid metabolic genes are predominantly confined to the posterior eyecup.

Transcriptomic similarities between homozygous Tmem135 mutant eyecups and AMD donor eyes. It is well accepted that dysfunction within the RPE is implicated as a key pathobiological mechanism in $\mathrm{AMD}^{13-15}$. Since the ocular phenotype of Tmem $135^{\text {FUNO25/FUN025 }}$ mice shares similarities with AMD including the presence of subretinal immune cells, neuroinflammation and RPE autofluorescence ${ }^{3,16}$, we were interested in

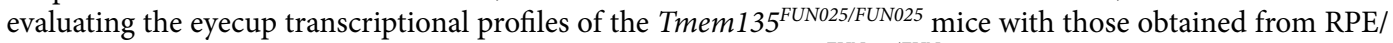

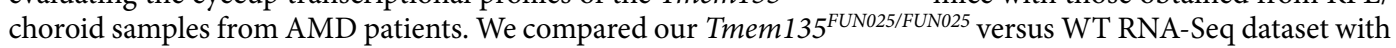
previously-published integrated microarray and RNA-Seq datasets (GSE29801 and GSE135092) using RPE/choroid samples from AMD patients ${ }^{17-19}$. There were similar genes affected due to the Tmem 135 mutation and AMD (Table 2), including 16 upregulated and 9 downregulated genes in common between the Tmem135 $5^{\text {FNO25/FUNO25 }}$ relative to WT RNA-Seq dataset and GSE29801 and GSE135092 datasets (Table 2 and Fig. 7A). In the GSE29801 

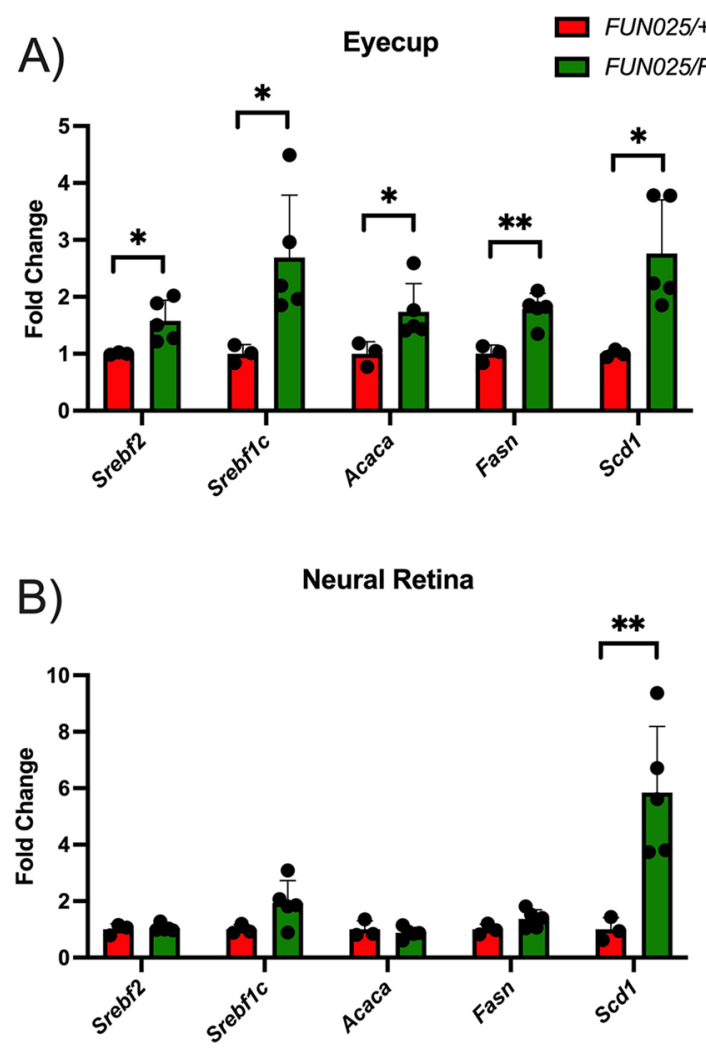

Figure 6. Lipid Metabolic Gene Expression in Eyecup and Neural Retinas of 2.5-month-old Heterozygous and Homozygous Tmem135 Mutant Mice. Quantitative PCR analysis of lipid metabolic transcription factors (Srebf2 and Srebf1c) and lipid synthesis genes (Acaca, Fasn, and Scd1) in the (A) eyecups and (B) neural retinas of 2.5-month-old male Tmem $135^{\text {FUN025/+ }}$ (FUN025/+) and Tmem135 FUN025/FUN025 (FUN025/FUN025) mice. Numbers in brackets denote number of mice used in experiment. Each dot represents each individual sample. * and ${ }^{* *}$ indicates a $P<0.05$ and $P<0.01$ significance by Student's T-test.

\begin{tabular}{|l|l|l|}
\hline \multirow{2}{*}{ Stage of AMD } & Genes in Common Between Tmem135 & FUn025/FUN025 eyecups and AMD-afflicted RPE/choroid samples \\
\cline { 2 - 3 } Mixed & Downregulated & Upregulated \\
\hline Intermediate & $\begin{array}{l}\text { Samd11, Gata2, Ddt, Cyb5a, Des, } \\
\text { Procr, Galnt9, Arpp21, Ramp2 }\end{array}$ & $\begin{array}{l}\text { Chrnb4, Srebf1, Fasn, Acat2, Fads1, Fads2, Col8a1, Lss, Cd109, Bmp8b, } \\
\text { Hmgcs1, Msmo1, Arhgap32, Mpv17l, Eif5a2, Insig1 }\end{array}$ \\
\hline Advanced & Cyb5a & Chrnb4, Srebf1, Fasn, Acat2, Col8a1, Lss \\
\hline
\end{tabular}

Table 2. Summary of significantly-affected genes in common between Tmem $135^{\text {FUN025/FUN025 }}$ versus WT eyecup RNAseq and GSE29801 and GSE135092 datasets.

dataset, some AMD RPE/choroid samples were classified as having an intermediate and advanced AMD

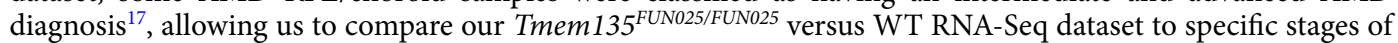
AMD. Six upegulated genes [cholinergic receptor nicotinic beta 4 subunit (Chrnb4), Srebf1, Fasn, acetyl-CoA acetyltransferase 2 (Acat2), collagen type VIII alpha 1 chain (Col8a1), and lanosterol synthase (Lss)] and one

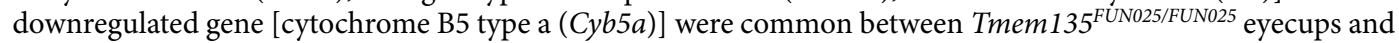
intermediate AMD (Table 2 and Fig. 7A). Furthermore, two upregulated genes (Srebf1 and Lss) and two downregulated genes [GATA binding protein 2 (Gata2) and Cyb5a] were common between Tmem135 FUN025/FUN025 eyecups and advanced AMD (Table 2 and Fig. 7A). We performed a gene over-representation analysis on the genes in Table 2 and found significant enrichment (FDR $<0.01)$ of multiple lipid metabolic pathways including cholesterol metabolism, sterol biosynthetic process, sterol metabolic process, lipid biosynthetic process, fatty acid biosynthetic process, and fatty acid metabolism (Table 3). We also investigated the network signaling path-

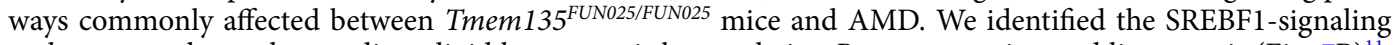
pathway, a pathway that mediates lipid homeostasis by regulating Pparg expression and lipogenesis (Fig. 7B) ${ }^{11}$, in common between Tmem $135^{\text {FUN025/FUN025 }}$ eyecups and AMD. Similarities between the Tmem135 ${ }^{\text {FUNO25/FUNO25 }}$ mice and AMD transcriptional profiles suggest the Tmem $135^{\text {FUNO25/FUN025 }}$ mouse may serve as a valuable model to interrogate the role of lipid metabolism on AMD-like pathology development. 
A)
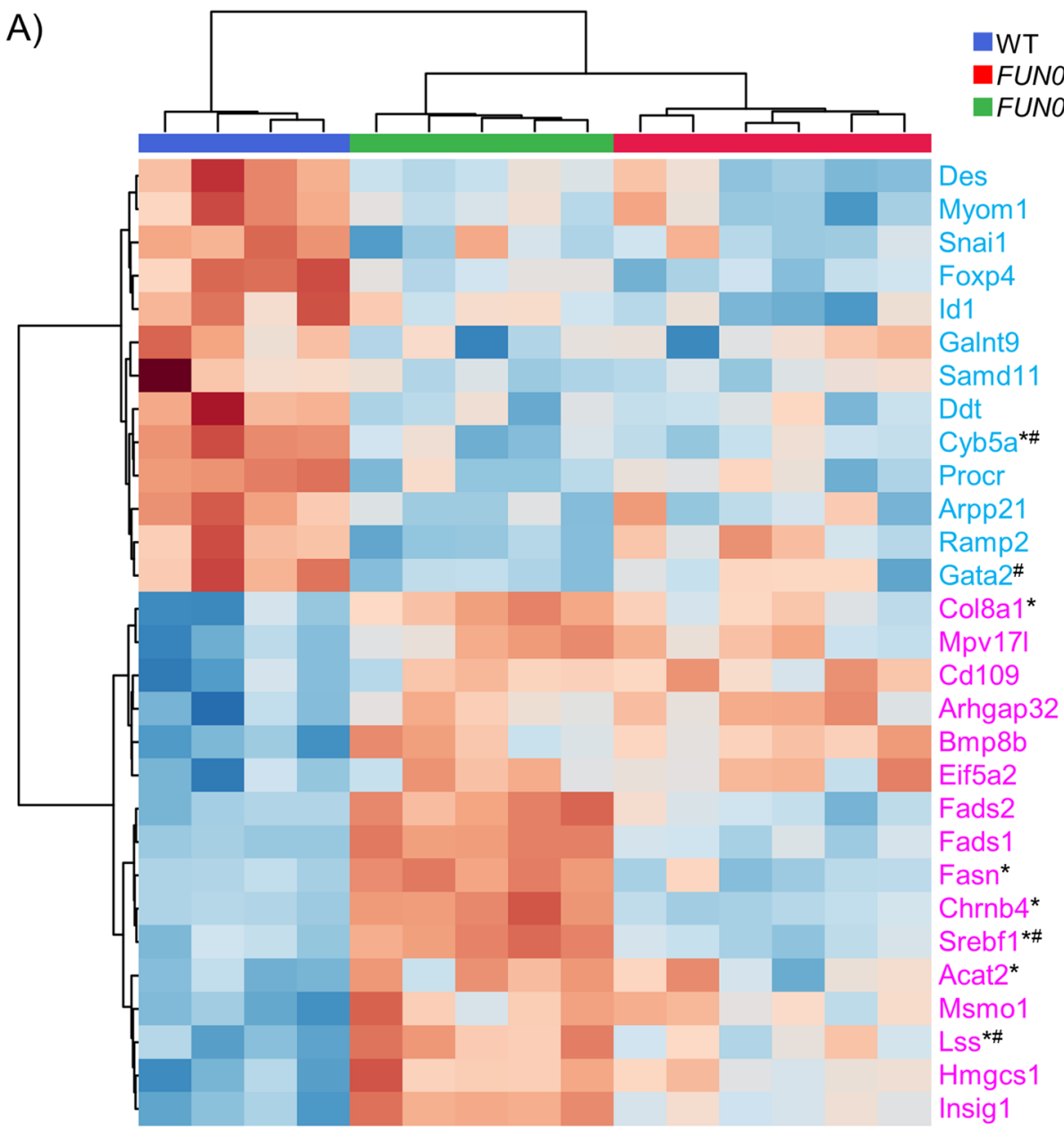

B)

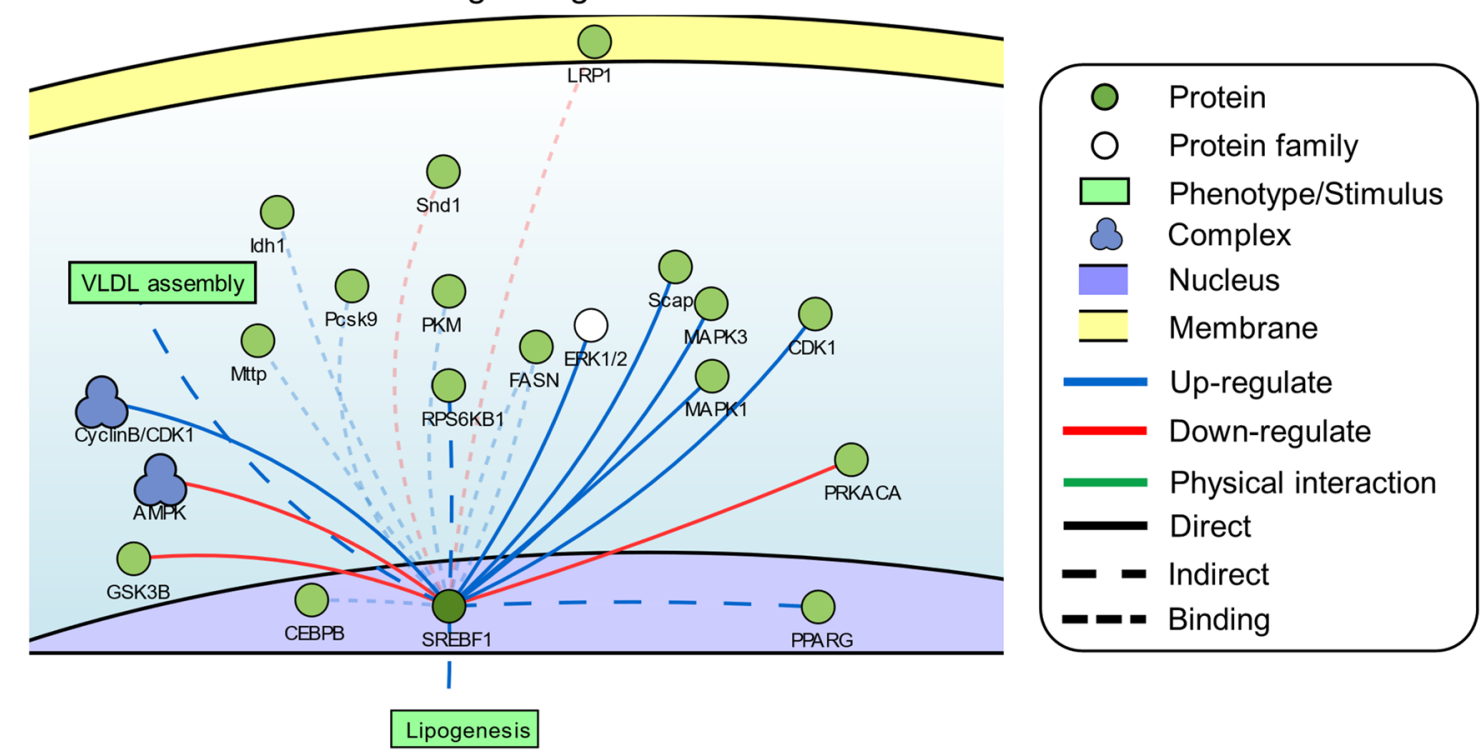

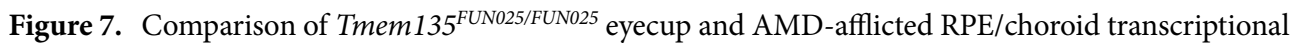
profiles. (A) Heatmap showing the significantly altered genes from Tmem $135^{\text {FUN025/FUN025 }}$ vs. WT which are affected in a similar direction (up [magenta] or down [cyan]) when compared with integrated expression analysis from microarray and RNA-Seq data (GSE29801 and GSE135092) from AMD patients. Genes which are affected in *intermediate and "advanced stage of AMD are also highlighted. (B) Signalling network highlights interactions of Sterol Regulatory Element Binding Transcription Factor 1 (SREBF1). Network generated using the SIGnalling Network Open Resource (SIGNOR 2.0). 


\begin{tabular}{|l|l|l|}
\hline Description & Enrichment ratio & FDR \\
\hline WP4346: Cholesterol metabolism & 26.40 & $7.41 \times 10^{-7}$ \\
\hline GO:0016126: Sterol biosynthetic process & 37.99 & $1.80 \times 10^{-3}$ \\
\hline GO:0016125: Sterol metabolic process & 18.16 & $1.81 \times 10^{-3}$ \\
\hline GO:0008610: Lipid biosynthetic process & 7.16 & $1.81 \times 10^{-3}$ \\
\hline GO:0006633: Fatty acid biosynthetic process & 17.35 & $1.81 \times 10^{-3}$ \\
\hline mmu01212: Fatty acid metabolism & 21.95 & $9.41 \times 10^{-3}$ \\
\hline
\end{tabular}

Table 3. Pathway analysis using overrepresentation analysis (ORA) on genes significantly-affected in both

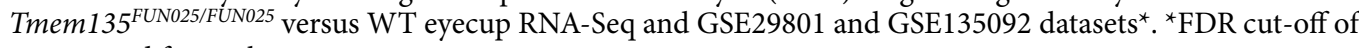
0.01 is used for analysis.

\section{Discussion}

Our current study builds on previous work that identified TMEM135 as a key protein involved in mitochondrial dynamics ${ }^{3,7,20,21}$, metabolic regulation ${ }^{8}$ and oxidative stress in mice ${ }^{3}$. However, the molecular function of TMEM135 remains elusive. In our study, we evaluated the global transcriptome of posterior eyecups to understand how a Tmem 135 mutation disrupts retinal homeostasis in mice. Both Tmem $135^{\text {FUNO25/+ }}$ and Tmem $135^{\text {FUNO25/FUN025 }}$

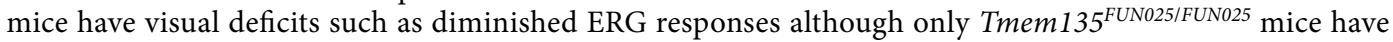
structural abnormalities including photoreceptor degeneration and subretinal immune cell infiltration. We also found the eyecup transcriptional profiles of both Tmem $135^{\mathrm{FUNO25/+}}$ and Tmem $135^{\mathrm{FUNO25/FUNO25}}$ mice deviate from WT controls, indicating one allele of the Tmem 135 mutation is sufficient to disrupt murine retinal homeostasis. Together, we conclude Tmem 135 is a critical gene involved in ocular health.

Tmem 135 encodes a membrane-bound protein that can localize with mitochondria ${ }^{3,5}$, peroxisomes ${ }^{22}$, and lipid droplets $^{5}$. Our gene enrichment analysis highlighted significant downregulation of mitochondrial membrane and upregulation of lipid metabolism pathways in Tmem $135^{\text {FUN025/FUN025 }}$ eyecups. Mitochondria, peroxisomes, and lipid droplets interact with each other to maintain lipid metabolism within a cell ${ }^{23}$. In particular, cells depend on mitochondria for the complete breakdown of lipids through oxidative phosphorylation ${ }^{24}$. The mitochondria in Tmem $135^{\text {FUNO25/FUN025 RPE appear overly elongated }}{ }^{7}$ and correlates with increased levels of glycerol ${ }^{8}$, a building block of triglycerides. Triglycerides store fatty acids within the cell for consumption by mitochondria for energy utilization $^{25}$. It has been shown that mitochondria with close proximities to lipid droplets are overly elongated ${ }^{26}$, which could explain the mitochondrial morphological changes in Tmem135 $5^{\text {FUN025/FUN025 }}$ RPE. Thus, TMEM135 may have a role in lipid metabolism within the RPE, supporting other studies linking the function of TMEM135 to lipid metabolism ${ }^{5,6}$.

The RPE is critical for sustaining lipid homeostasis within the retina, digesting photoreceptor outer segments, ingesting choroid-derived lipoproteins, and transporting lipids out of the retina ${ }^{12}$. It is known that RPE cells express canonical pathways involved in lipid homeostasis including the sterol regulatory element-binding protein (SREBF) pathway in mice and humans ${ }^{27,28}$ but may rely on additional mechanisms as suggested by a lack of responsiveness of cholesterol-sensitive genes in wild-type mouse retinas after the consumption of dietary cholesterol $^{27}$. Through our analysis of differential genes that correlated with the number of Tmem 135 mutant alleles, we identified BCOR and IDH1 as potential players in RPE lipid homeostasis. BCOR is a co-repressor of BCL6 that mediates BCL6-mediated transcriptional repression ${ }^{10}$. There have been no published reports on a role for BCOR in lipid metabolism, but loss of BCL6 in the liver augments lipid catabolism, lessens highfat-diet induced hepatic steatosis, and reverses fatty acid breakdown and lipid accumulation induced by fasting in peroxisome proliferator activated receptor alpha (Ppara) knockout mice ${ }^{29}$. The decrease in Bcor in the Tmem 135 $5^{\mathrm{FUNO25/FUN025}}$ mouse eyecups may be in response to the increased lipid accumulation such as cholesterol and neutral lipids. IDH1 is an isocitrate dehydrogenase enzyme found in the cytoplasm as well as on peroxisomes that catalyzes the reversible oxidative decarboxylation of isocitrate to alpha-ketoglutarate ${ }^{30}$. Loss-of-function mutations in human IDH1 lead to increased monosaturated fatty acids and increased SCD expression in infil-

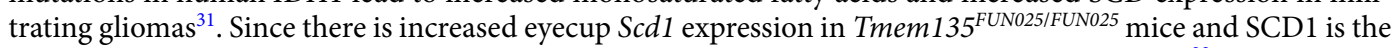
rate-limiting enzyme for the conversion of saturated fatty acids to monounsaturated fatty acids ${ }^{32}$, the increased Idh1 expression in Tmem $135^{\text {FUNO25/FUN025 }}$ mouse eyecups may occur to decrease the amount of monounsaturated

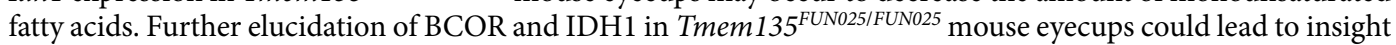
into lipid metabolism within RPE cells.

Dysregulation of lipid metabolism is strongly associated with AMD development and progression ${ }^{33}$. The association of dysregulated lipid metabolism and AMD comes from genetic and environmental studies. Genetic studies have linked variants with human apolipoprotein $\mathrm{E}(A P O E)$, hepatic lipase $(L I P C)$, cholesteryl ester transfer protein (CETP), lipoprotein lipase $(L P L)$, and ATP binding cassette subfamily A member 1 (ABCA1) with risk for $\mathrm{AMD}^{34-40}$. The roles of these genes in AMD development and progression are largely unknown. Furthermore, consumption of diets high in fat ${ }^{41-43}$, particularly those with high levels of omega- 6 , monounsaturated, polyunsaturated and trans unsaturated fatty acids ${ }^{44,45}$, increases the likelihood of developing AMD. In contrast, eating diets with a high concentration of fish oils containing omega- 3 fatty acids decreases the incidence of AMD ${ }^{44-47}$. How diets influence retinal homeostasis and modulate the risk for AMD is unclear. To understand the role of these epidemiological risk factors for AMD, it is imperative to study models in which we can gain new insight

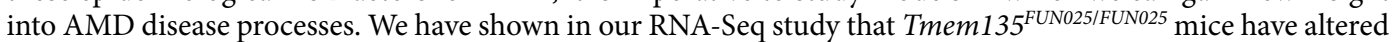
expression of lipid metabolic genes in their posterior eyecups and lipid accumulation in their RPE. Some of these 


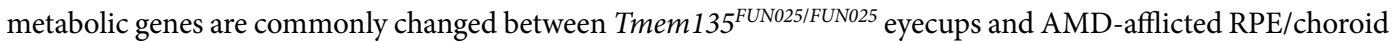

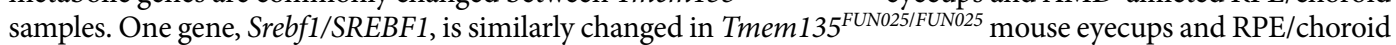
samples from human patients diagnosed with mixed, intermediate, and advanced stages of AMD. SREBF1 is a transcription factor that regulates the expression of sterol-regulated genes and controls cholesterol homeostasis ${ }^{48}$. SREBF1 also positively regulates the expression of lipogenesis genes including Fasn/FASN ${ }^{49}$ which is increased in both Tmem $135^{\text {FUNO25/FUN025 }}$ mouse eyecups and RPE/choroid samples from human patients diagnosed with mixed and intermediate AMD. Future studies on the role of SREBF1 in Tmem $135^{\text {FUN025/FUN025 retinas may provide }}$ clues on its role in AMD development and progression.

Aging is a significant risk factor for a number of diseases including AMD and Alzheimer's disease, but how aging contributes to these diseases is unknown. One strategy to better understand the role of aging in disease development is to study mutant mice with accelerated aging phenotypes and uncover the genetic causes of their accelerated aging phenotypes. Utilizing this strategy, we identified Tmem 135 as a gene involved in the aging process in the retina ${ }^{3}$. In the current study, we undertook an RNA-Seq study to further investigate the consequences of the Tmem 135 mutation on retinal homeostasis. We found that mutant Tmem135 mice display significant transcriptomic changes but one significant pathway that contained a number of differentially-expressed genes is lipid metabolism. Some of these genes are also altered in transcriptomic profiles of AMD-diagnosed RPE/ choroid specimens. Studying Tmem 135 mutant mice may provide insight into how aging effects lipid metabolism and contributes to diseases such as AMD.

\section{Methods}

Mice. Generation of Tmem $135^{\mathrm{FUNO} 25 /{ }^{+}}$and Tmem $135^{\text {FUNO25/FUN025 }}$ mice on the C57BL/6 J background was previously described ${ }^{3,7}$. WT mice on the C57BL/6 J background were used as controls for these experiments. All animals were housed in the same animal facility at the University of Wisconsin-Madison under the same environmental conditions. Both male and female mice that were 2.5 months, 3 months and 12 months of age were used in this study. Details of mice used in each figure of this study are provided in Supplemental Table 1. No mice used in this study were positive for the Ped $6 b^{r d 1}$ or the $C r b 1^{r d 8}$ mutation ${ }^{50,51}$. All experiments performed in this study were in accordance with the National Institute of Health Guide for the Care and Use of Laboratory Animals and authorized by the Animal Care and Use Committee at the University of Wisconsin-Madison. The results and methods in this study are reported in accordance to the ARRIVE guidelines.

Histology. Eyes were fixed in 2\% PFA and $2 \%$ glutaraldehyde overnight at $4{ }^{\circ} \mathrm{C}$ and submitted to the University of Wisconsin-Madison's Translational Research Initiatives in Pathology (TRIP) core for paraffin processing. Sections were stained with hematoxylin and eosin (H\&E) to visualize retinal layers and imaged using an Axio Imager 2 microscope (Carl Zeiss MicroImaging, White Plains, NY) at a 40X magnification. Outer nuclear layer (ONL) and inner nuclear layer (INL) thickness were measured using ImageJ (NIH, Bethesda, MD) on a single section from each eye. Measurements were started from the optic nerve head and taken at seven consecutive 300 micron intervals.

Electroretinography (ERG). Mice were dark-adapted overnight before ERG recording and then anesthetized with a cocktail of ketamine $(80 \mathrm{mg} / \mathrm{kg})$ and xylazine $(16 \mathrm{mg} / \mathrm{kg})$ diluted in phosphate buffered saline (PBS). Pupils were dilated with $1 \%$ tropicamide, and a drop of sterile $2.5 \%$ hypromellose ophthalmic solution (Goniovisc, HUB pharmaceuticals LLC, CA) was applied to the cornea for recording electrodes. ERGs were recorded using the Espion system (Diagnosys LLC, MA). Scotopic recordings were obtained from dark-adapted animals, and the eyes were exposed to a sequential increment of flash intensities $\left(0.1\right.$ to $\left.30 \mathrm{~cd} . \mathrm{s} / \mathrm{m}^{2}\right)$ for $300 \mathrm{~ms}$ with a $2 \mathrm{~s}$ interval between the flashes. For the acquisition of c-wave, the eyes were flashed with light intensities of 2.5 and $25 \mathrm{~cd} . \mathrm{s} / \mathrm{m}^{2}$ for $4 \mathrm{~s}$. Photopic recordings were performed after the light adaptation of mice at $30 \mathrm{~cd} . \mathrm{m}^{2}$ background and during the photopic recordings. The flash intensities used for the photopic recordings were $0.78,1,2.25,5$ and $10 \mathrm{~cd} . \mathrm{s} / \mathrm{m}^{2}$. Only the $10 \mathrm{~cd} . \mathrm{s} / \mathrm{m}^{2}$ recordings were used in this study. ERG components were measured using the Espion software (Diagnosys LLC, MA) and analyzed using Origin2020 (OriginLab Corp., MA).

Bulk-RNA sequencing. Posterior eyecups without the neural retina were collected and pooled from indi-

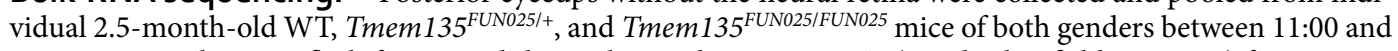
1:00 PM. Samples were flash frozen and then submitted to GENEWIZ (South Plainfield, NJ, USA) for processing. Total RNA was extracted from eyecups with an RNeasy Plus Universal Mini kit (Qiagen, Hilden, Germany) following the Manufacturer's instructions. Total RNA samples were quantified using a Qubit 2.0 Fluorometer (Life Technologies, Carlsbad, CA, USA). RNA integrity was examined using a TapeStation 4200 (Agilent Technologies, Palo Alto, CA, USA). RNA sequencing libraries were prepared using the NEBNext Ultra RNA Library Prep Kit for Illumina (NEB, Ipswich, MA, USA) following manufacturer's instructions. Briefly, messenger RNAs were first enriched with Oligo(dT) beads. Enriched mRNAs were fragmented for $15 \mathrm{~min}$ at $94{ }^{\circ} \mathrm{C}$. First strand and second strand cDNAs were subsequently synthesized. cDNA fragments were end repaired and adenylated at 3'ends, and universal adapters were ligated to cDNA fragments, followed by index addition and library enrichment by limited-cycle PCR. The sequencing libraries were validated on the Agilent TapeStation and quantified by using Qubit 2.0 Fluorometer as well as by quantitative PCR (KAPA Biosystems, Wilmington, MA, USA). The sequencing libraries were clustered on a flowcell. After clustering, the flowcell was loaded on the Illumina HiSeq instrument (4000 or equivalent) according to manufacturer's instructions. The samples were sequenced using a $2 \times 150$ bp Paired End (PE) configuration. Image analysis and base calling were conducted by the HiSeq Control Software (HCS). Raw sequence data (.bcl files) generated from Illumina HiSeq were converted into fastq files 
and de-multiplexed using Illumina's bcl2fastq 2.17 software. The RNA-Seq raw sequence files from this study are available on the Gene Expression Omnibus (GEO), accession number GSE184160.

RNA sequencing analysis. Gene expression read counts were analysed using NetworkAnalyst $3 \cdot 0^{52} . M$. musculus organism was selected with bulk sequencing analysis workflow. Quality control step involved filtering genes with very high variance across samples. Genes were ranked based on variance and those genes which ranked in the bottom $15 \%$ of the percentile were filtered out. Low abundance genes below a threshold were also filtered out. Data was normalized using $\log 2$ counts per million normalization method. Differential gene expression analysis was performed using EdgeR ${ }^{53}$. Gene set enrichment analysis was performed using WebGestalt ${ }^{54}$ and different functional databases including Gene Ontology, KEGG, and WikiPathways were used for analysis. To further restrict the number of gene sets due to overlap of the genes, affinity cluster algorithm ${ }^{55}$ was applied. Heatmaps were generated on auto-scaled normalized gene expression data using Euclidean distance measure and ward clustering algorithm using MetaboAnalyst $4.0^{56}$. Signalling pathway analysis was conducted on differentially expressed genes using the SIGNOR 2.0 database ${ }^{57}$. A comparison of differentially expressed genes in different groups with human age-related macular degeneration samples was conducted by comparing the fold change (up or down) of differentially expressed genes identified from integrated microarray (GSE29801 dataset) ${ }^{17}$ and RNA-seq analysis (GSE135092 dataset) ${ }^{18}$ of AMD human donor eyes compared to non-AMD control donor eyes. AMD donor eyes used to generate the GSE29801 dataset were independently classified into distinct AMD categories based on the Age-Related Eye Disease Study (AREDS) and Rotterdam grading scales ${ }^{17}$ whereas AMD donor eyes graded as having category 4 disease were used to produce the GSE135092 dataset ${ }^{18}$. In total, the GSE29801 dataset was comprised of expression data from 31 control eyes and 37 AMD-afflicted eyes ${ }^{17}$ while the GSE135092 dataset contained transcriptomic data from 106 control eyes and 23 AMD eyes ${ }^{18}$.

Cholesterol measurement. Mice were perfused with PBS prior to enucleation of eyes. Eyes were processed to remove the anterior segment, lens, and neural retina. The remaining posterior eyecups were flash frozen with liquid nitrogen before processing. Frozen eyecups were homogenized using $80 \mathrm{ul}$ of RIPA lysis and extraction buffer (\#PI89901, VWR, Radnor, PA) with ethylenediaminetetraacetic acid (EDTA)-free protease inhibitors (\#11836170001, Sigma-Aldrich, St. Louis, MO). Cholesterol was measured using a colorimetric Total Cholesterol Assay Kit (\#STA-384, Cell Biolabs, San Diego, CA). Cholesterol values were normalized to the weight of the individual eyecup.

RPE flat mount immunofluorescence. Eyes were removed and processed for RPE flat mounts using a previously described protocol$^{7}$. RPE flat mounts were incubated with a rabbit polyclonal zonula occludens-1 antibody (\#40-2200, Thermo Fisher Scientific, Waltham, MA) and HCS Lipidtox Red Neutral Lipid Stain (\#H34476, Thermo Fisher Scientific, Waltham, MA) overnight at $4{ }^{\circ} \mathrm{C}$. Then, the eyecups were incubated with an Alexa Fluor 488 conjugated donkey anti-rabbit IgG (\#A-21206, Thermo Fisher Scientific, Waltham, MA) and 4',6-Diamidine-2'-phenylindole dihydrochloride (DAPI) (\#D9542, Sigma Aldrich, St. Louis, MO) for two hours. RPE flat mounts were imaged with a Nikon A1RS confocal microscope at a $20 \times$ magnification.

Quantitative real-time PCR. Eyecups and neural retinas from mice were isolated after PBS perfusion, flash frozen, and kept at $-80^{\circ} \mathrm{C}$. RNA was isolated using a RNeasy lipid tissue mini kit (\#74804, Qiagen, Germantown, MD). Concentrations of RNA were calculated using a Nanodrop 2000 UV-Vis Spectrophotometer (Thermo Fisher Scientific, Waltham, MA). Equal RNA concentrations were aliquoted to make cDNA using an Oligo d(T)18 primer (\#S1316S, NEB, Ipswich, MA) and the ProtoScript II Reverse Transcriptase (\#M0368L, NEB, Ipswich, MA). Triplicate reactions for each gene was run in the Roche Lightcycler 480 system using cDNA, $200 \mathrm{nmol} / \mathrm{L}$ of each primer, and Lightcycler 480 SYBR Green I Supermix (\#507203180, Roche). Primer sequences used in this study are described in Supplemental Table 2. Relative expression was normalized to the ribosomal protein lateral stalk subunit P0 (Rlplo) using the quantitative $2^{-\Delta \Delta C T}$ method $^{58}$.

Statistical analysis. Statistics were performed using GraphPad Prism software (GraphPad Software, Inc., San Diego, CA). Details of statistical tests are provided in figure legends.

Received: 15 September 2021; Accepted: 28 December 2021

Published online: 14 January 2022

\section{References}

1. Van Cauwenberghe, C., Van Broeckhoven, C. \& Sleegers, K. The genetic landscape of Alzheimer disease: Clinical implications and perspectives. Genet. Med. 18, 421-430. https://doi.org/10.1038/gim.2015.117 (2016).

2. Fritsche, L. G. et al. Age-related macular degeneration: Genetics and biology coming together. Annu. Rev. Genomics Hum. Genet. 15, 151-171. https://doi.org/10.1146/annurev-genom-090413-025610 (2014).

3. Lee, W. H. et al. Mouse Tmem 135 mutation reveals a mechanism involving mitochondrial dynamics that leads to age-dependent retinal pathologies. Elife 5, 1-29. https://doi.org/10.7554/eLife.19264 (2016).

4. Žárský, V. \& Doležal, P. Evolution of the Tim17 protein family. Biol Direct 11, 1-13. https://doi.org/10.1186/s13062-016-0157-y (2016).

5. Exil, V. J. et al. Stressed-induced TMEM135 protein is part of a conserved genetic network involved in fat storage and longevity regulation in Caenorhabditis elegans. PLoS ONE 5, 1-11. https://doi.org/10.1371/journal.pone.0014228 (2010). 
6. Maharjan, Y. et al. TMEM135 regulates primary ciliogenesis through modulation of intracellular cholesterol distribution. EMBO Rep. 21, 1-16. https://doi.org/10.15252/embr.201948901 (2020).

7. Landowski, M. et al. Modulation of Tmem 135 leads to retinal pigmented epithelium pathologies in mice. Invest. Ophthalmol. Vis. Sci. 61, 16. https://doi.org/10.1167/iovs.61.12.16 (2020).

8. Lee, W. H. et al. Metabolic alterations caused by the mutation and overexpression of the Tmem 135 gene. Exp. Biol. Med. (Maywood) https://doi.org/10.1177/1535370220932856 (2020).

9. Tilokani, L., Nagashima, S., Paupe, V. \& Prudent, J. Mitochondrial dynamics: Overview of molecular mechanisms. Essays Biochem. 62, 341-360. https://doi.org/10.1042/EBC20170104 (2018).

10. Astolfi, A. et al. BCOR involvement in cancer. Epigenomics 11, 835-855. https://doi.org/10.2217/epi-2018-0195 (2019).

11. Raghow, R., Yellaturu, C., Deng, X., Park, E. A. \& Elam, M. B. SREBPs: The crossroads of physiological and pathological lipid homeostasis. Trends Endocrinol. Metab. 19, 65-73. https://doi.org/10.1016/j.tem.2007.10.009 (2008).

12. Lakkaraju, A. et al. The cell biology of the retinal pigment epithelium. Prog. Retin. Eye Res. https://doi.org/10.1016/j.preteyeres. 2020.100846 (2020).

13. Fisher, C. R. \& Ferrington, D. A. Perspective on AMD pathobiology: A bioenergetic crisis in the RPE. Invest. Ophthalmol. Vis. Sci. 59, AMD41-AMD47. https://doi.org/10.1167/iovs.18-24289 (2018).

14. George, S. M., Lu, F., Rao, M., Leach, L. L. \& Gross, J. M. The retinal pigment epithelium: Development, injury responses, and regenerative potential in mammalian and non-mammalian systems. Prog. Retin. Eye Res. https://doi.org/10.1016/j.preteyeres.2021. 100969 (2021).

15. Somasundaran, S., Constable, I. J., Mellough, C. B. \& Carvalho, L. S. Retinal pigment epithelium and age-related macular degeneration: A review of major disease mechanisms. Clin. Exp. Ophthalmol. 48, 1043-1056. https://doi.org/10.1111/ceo.13834 (2020).

16. Copland, D. A., Theodoropoulou, S., Liu, J. \& Dick, A. D. A Perspective of AMD Through the Eyes of Immunology. Invest. Ophthalmol. Vis. Sci. 59, AMD83-AMD92. https://doi.org/10.1167/iovs.18-23893 (2018).

17. Newman, A. M. et al. Systems-level analysis of age-related macular degeneration reveals global biomarkers and phenotype-specific functional networks. Genome Med. 4, 16. https://doi.org/10.1186/gm315 (2012).

18. Orozco, L. D. et al. Integration of eQTL and a single-cell atlas in the human eye identifies causal genes for age-related macular degeneration. Cell. Rep. 30, 1246-1259.e1246. https://doi.org/10.1016/j.celrep.2019.12.082 (2020).

19. Dhirachaikulpanich, D., Li, X., Porter, L. F. \& Paraoan, L. Integrated Microarray and RNAseq transcriptomic analysis of retinal pigment epithelium/choroid in age-related macular degeneration. Front. Cell. Dev. Biol 8, 808. https://doi.org/10.3389/fcell.2020. 00808 (2020).

20. Lewis, S. A. et al. The effect of Tmem 135 overexpression on the mouse heart. PLoS ONE 13, 1-29. https://doi.org/10.1371/journ al.pone.0201986 (2018).

21. Muench, N. A. et al. The influence of mitochondrial dynamics and function on retinal ganglion cell susceptibility in optic nerve disease. Cells https://doi.org/10.3390/cells10071593 (2021).

22. Maharjan, Y. et al. TMEM135 regulates primary ciliogenesis through modulation of intracellular cholesterol distribution. EMBO Rep. https://doi.org/10.15252/embr.201948901 (2020).

23. Kohlwein, S. D., Veenhuis, M. \& van der Klei, I. J. Lipid droplets and peroxisomes: Key players in cellular lipid homeostasis or a matter of fat-store 'em up or burn 'em down. Genetics 193, 1-50. https://doi.org/10.1534/genetics.112.143362 (2013).

24. Aon, M. A., Bhatt, N. \& Cortassa, S. C. Mitochondrial and cellular mechanisms for managing lipid excess. Front. Physiol. 5, 282. https://doi.org/10.3389/fphys.2014.00282 (2014).

25. Alves-Bezerra, M. \& Cohen, D. E. Triglyceride metabolism in the liver. Compr. Physiol. 8, 1-8. https://doi.org/10.1002/cphy.c1700 12 (2017).

26. Benador, I. Y. et al. Mitochondria bound to lipid droplets have unique bioenergetics, composition, and dynamics that support lipid droplet expansion. Cell. Metab. 27, 869-885.e866. https://doi.org/10.1016/j.cmet.2018.03.003 (2018).

27. Zheng, W., Mast, N., Saadane, A. \& Pikuleva, I. A. Pathways of cholesterol homeostasis in mouse retina responsive to dietary and pharmacologic treatments. J. Lipid Res. 56, 81-97. https://doi.org/10.1194/jlr.M053439 (2015).

28. Zheng, W. et al. Spatial distribution of the pathways of cholesterol homeostasis in human retina. PLoS ONE 7, e37926. https://doi. org/10.1371/journal.pone.0037926 (2012).

29. Sommars, M. A. et al. Dynamic repression by BCL6 controls the genome-wide liver response to fasting and steatosis. Elife https:// doi.org/10.7554/eLife.43922 (2019).

30. Bogdanovic, E. IDH1, lipid metabolism and cancer: Shedding new light on old ideas. Biochim. Biophys. Acta 1850, 1781-1785. https://doi.org/10.1016/j.bbagen.2015.04.014 (2015).

31. Lita, A. et al. IDH1 mutations induce organelle defects via dysregulated phospholipids. Nat. Commun. 12, 614. https://doi.org/10. 1038/s41467-020-20752-6 (2021).

32. Flowers, M. T. \& Ntambi, J. M. Role of stearoyl-coenzyme A desaturase in regulating lipid metabolism. Curr. Opin. Lipidol. 19, 248-256. https://doi.org/10.1097/MOL.0b013e3282f9b54d (2008).

33. Miller, J. W. Age-related macular degeneration revisited-piecing the puzzle: The LXIX Edward Jackson memorial lecture. Am J Ophthalmol 155, 1-35. https://doi.org/10.1016/j.ajo.2012.10.018 (2013).

34. Klaver, C. C. et al. Genetic association of apolipoprotein E with age-related macular degeneration. Am. J. Hum. Genet. 63, $200-206$. https://doi.org/10.1086/301901 (1998).

35. Schmidt, S. et al. Association of the apolipoprotein E gene with age-related macular degeneration: Possible effect modification by family history, age, and gender. Mol. Vis. 6, 287-293 (2000).

36. Zareparsi, S. et al. Association of apolipoprotein $\mathrm{E}$ alleles with susceptibility to age-related macular degeneration in a large cohort from a single center. Invest. Ophthalmol. Vis. Sci. 45, 1306-1310. https://doi.org/10.1167/iovs.03-1253 (2004).

37. McKay, G. J. et al. Evidence of association of APOE with age-related macular degeneration: A pooled analysis of 15 studies. Hum. Mutat. 32, 1407-1416. https://doi.org/10.1002/humu.21577 (2011).

38. Chen, W. et al. Genetic variants near TIMP3 and high-density lipoprotein-associated loci influence susceptibility to age-related macular degeneration. Proc. Natl. Acad. Sci. U. S. A. 107, 7401-7406. https://doi.org/10.1073/pnas.0912702107 (2010).

39. Neale, B. M. et al. Genome-wide association study of advanced age-related macular degeneration identifies a role of the hepatic lipase gene (LIPC). Proc. Natl. Acad. Sci. U. S. A. 107, 7395-7400. https://doi.org/10.1073/pnas.0912019107 (2010).

40. Wang, Y. F. et al. CETP/LPL/LIPC gene polymorphisms and susceptibility to age-related macular degeneration. Sci. Rep. 5, 15711. https://doi.org/10.1038/srep15711 (2015).

41. Clemons, T. E., Milton, R. C., Klein, R., Seddon, J. M. \& Ferris, F. L. 3rd. Risk factors for the incidence of advanced age-related macular degeneration in the age-related eye disease study (AREDS) AREDS report no. 19. Ophthalmology 112, 533-539 (2005).

42. Heiba, I. M., Elston, R. C., Klein, B. E. \& Klein, R. Sibling correlations and segregation analysis of age-related maculopathy: The Beaver Dam Eye Study. Genet. Epidemiol. 11, 51-67 (1994).

43. Vingerling, J. R., Klaver, C. C., Hofman, A. \& de Jong, P. T. Epidemiology of age-related maculopathy. Epidemiol. Rev. 17, 347-360 (1995).

44. Seddon, J. M. et al. Dietary fat and risk for advanced age-related macular degeneration. Arch. Ophthalmol. 119, 1191-1199. https:// doi.org/10.1001/archopht.119.8.1191 (2001).

45. Seddon, J. M., Cote, J. \& Rosner, B. Progression of age-related macular degeneration: Association with dietary fat, transunsaturated fat, nuts, and fish intake. Arch. Ophthalmol. 121, 1728-1737. https://doi.org/10.1001/archopht.121.12.1728 (2003). 
46. Cho, E. et al. Prospective study of dietary fat and the risk of age-related macular degeneration. Am. J. Clin. Nutr. 73, 209-218. https://doi.org/10.1093/ajcn/73.2.209 (2001).

47. Seddon, J. M., George, S. \& Rosner, B. Cigarette smoking, fish consumption, omega-3 fatty acid intake, and associations with age-related macular degeneration: The US Twin Study of Age-Related Macular Degeneration. Arch. Ophthalmol. 124, 995-1001. https://doi.org/10.1001/archopht.124.7.995 (2006).

48. Osborne, T. F. CREating a SCAP-less liver keeps SREBPs pinned in the ER membrane and prevents increased lipid synthesis in response to low cholesterol and high insulin. Genes Dev. 15, 1873-1878. https://doi.org/10.1101/gad.916601 (2001).

49. Horton, J. D., Goldstein, J. L. \& Brown, M. S. SREBPs: Activators of the complete program of cholesterol and fatty acid synthesis in the liver. J. Clin. Invest. 109, 1125-1131. https://doi.org/10.1172/JCI15593 (2002).

50. Pittler, S. J. \& Baehr, W. Identification of a nonsense mutation in the rod photoreceptor cGMP phosphodiesterase beta-subunit gene of the rd mouse. Proc. Natl. Acad. Sci U. S. A. 88, 8322-8326. https://doi.org/10.1073/pnas.88.19.8322 (1991).

51. Mattapallil, M. J. et al. The Rd8 mutation of the Crb1 gene is present in vendor lines of C57BL/6N mice and embryonic stem cells, and confounds ocular induced mutant phenotypes. Invest. Ophthalmol. Vis. Sci. 53, 2921-2927. https://doi.org/10.1167/iovs.129662 (2012).

52. Zhou, G. et al. NetworkAnalyst 3.0: A visual analytics platform for comprehensive gene expression profiling and meta-analysis. Nucleic Acids Res. 47, W234-W241. https://doi.org/10.1093/nar/gkz240 (2019).

53. Robinson, M. D., McCarthy, D. J. \& Smyth, G. K. edgeR: A Bioconductor package for differential expression analysis of digital gene expression data. Bioinformatics 26, 139-140. https://doi.org/10.1093/bioinformatics/btp616 (2010).

54. Liao, Y., Wang, J., Jaehnig, E. J., Shi, Z. \& Zhang, B. WebGestalt 2019: Gene set analysis toolkit with revamped UIs and APIs. Nucleic Acids Res 47, W199-W205. https://doi.org/10.1093/nar/gkz401 (2019).

55. Frey, B. J. \& Dueck, D. Clustering by passing messages between data points. Science 315, 972-976. https://doi.org/10.1126/scien ce.1136800 (2007).

56. Chong, J. et al. MetaboAnalyst 4.0: Towards more transparent and integrative metabolomics analysis. Nucleic Acids Res. 46, W486-W494. https://doi.org/10.1093/nar/gky310 (2018).

57. Licata, L. et al. SIGNOR 2.0, the SIGnaling network open resource 2.0: 2019 update. Nucleic Acids Res. 48, D504-D510. https:// doi.org/10.1093/nar/gkz949 (2020).

58. Livak, K. J. \& Schmittgen, T. D. Analysis of relative gene expression data using real-time quantitative PCR and the 2(-Delta Delta $\mathrm{C}(\mathrm{T})$ ) method. Methods 25, 402-408 (2001).

\section{Acknowledgements}

We would like to thank Satoshi Kinoshita and the University of Wisconsin Translational Research Initiatives in Pathology laboratory (TRIP), supported by the University of Wisconsin Department of Pathology and Laboratory Medicine, UWCCC (P30 CA014520) and the Office of the Director, National Institutes of Health (S10OD023526) for the use of their services. Grants: NIH R01 EY022086, Timothy William Trout Chairmanship, NIH T32EY027721, NIH F32EY032766, NIH P30 EY016665 and Unrestricted Grant from Research to Prevent Blindness, Inc.

\section{Author contributions}

M.L., V.J.B. and A.I. have made substantial contributions to the design of the work. M.L., V.J.B., T.T., S.G., P.K.S., B.R.P., and A.I. contributed to acquisition and analysis of work. M.L., V.J.B., S.I., and A.I. have drafted the manuscript. All authors have reviewed the manuscript.

\section{Competing interests}

The authors declare no competing interests.

\section{Additional information}

Supplementary Information The online version contains supplementary material available at https:/doi.org/ 10.1038/s41598-021-04644-3.

Correspondence and requests for materials should be addressed to A.I.

Reprints and permissions information is available at www.nature.com/reprints.

Publisher's note Springer Nature remains neutral with regard to jurisdictional claims in published maps and institutional affiliations.

(c) (i) Open Access This article is licensed under a Creative Commons Attribution 4.0 International License, which permits use, sharing, adaptation, distribution and reproduction in any medium or format, as long as you give appropriate credit to the original author(s) and the source, provide a link to the Creative Commons licence, and indicate if changes were made. The images or other third party material in this article are included in the article's Creative Commons licence, unless indicated otherwise in a credit line to the material. If material is not included in the article's Creative Commons licence and your intended use is not permitted by statutory regulation or exceeds the permitted use, you will need to obtain permission directly from the copyright holder. To view a copy of this licence, visit http://creativecommons.org/licenses/by/4.0/.

(c) The Author(s) 2022 